\title{
A template-proximal RNA paired element contributes to Saccharomyces cerevisiae telomerase activity
}

\author{
ANITA G. SETO, ${ }^{1}$ KFIR UMANSKY, ${ }^{3}$ YEHUDA TZFATI, ${ }^{3,4}$ ARTHUR J. ZAUG, ${ }^{1,2}$ ELIZABETH H. BLACKBURN, ${ }^{4}$ \\ and THOMAS R. CECH ${ }^{1,2}$ \\ ${ }^{1}$ Department of Chemistry and Biochemistry, and ${ }^{2}$ Howard Hughes Medical Institute, University of Colorado, \\ Boulder, Colorado 80309-0215, USA \\ ${ }^{3}$ Department of Genetics, Silberman Institute of Life Sciences, The Hebrew University of Jerusalem, 91904 Jerusalem, Israel \\ ${ }^{4}$ Department of Biochemistry and Biophysics, University of California, San Francisco, San Francisco, California 94143-2200, USA
}

\begin{abstract}
The ribonucleoprotein complex telomerase is critical for replenishing chromosome-end sequence during eukaryotic DNA replication. The template for the addition of telomeric repeats is provided by the RNA component of telomerase. However, in budding yeast, little is known about the structure and function of most of the remainder of the telomerase RNA. Here, we report the identification of a paired element located immediately 5' of the template region in the Saccharomyces cerevisiae telomerase RNA. Mutations disrupting or replacing the helical element showed that this structure, but not its exact nucleotide sequence, is important for telomerase function in vivo and in vitro. Biochemical characterization of a paired element mutant showed that the mutant generated longer products and incorporated noncognate nucleotides. Sequencing of in vivo synthesized telomeres from this mutant showed that DNA synthesis proceeded beyond the normal template. Thus, the $S$. cerevisiae element resembles a similar element found in Kluyveromyces budding yeasts with respect to a function in template boundary specification. In addition, the in vitro activity of the paired element mutant indicates that the RNA element has additional functions in enzyme processivity and in directing template usage by telomerase.
\end{abstract}

Keywords: Ribonucleoprotein; secondary structure; telomerase; template; yeast

\section{INTRODUCTION}

Telomerase, the chromosome end-replicating enzyme, contains an RNA subunit essential for its function (Greider and Blackburn 1987). The RNA provides the template for the synthesis of telomeric repeat sequences by the catalytic reverse transcriptase (Blackburn 2000). Telomerase is found in most eukaryotes, and the catalytic protein subunit (TERT) has been shown to have high sequence conservation among divergent organisms (Kelleher et al. 2002). The RNA subunit, on the other hand, shows little primary sequence conservation, even between closely related species. Furthermore, the length of telomerase RNAs varies greatly among eukaryotes: 150-200 nt for ciliated protozoa, 300-500 nt for vertebrates, and $>1 \mathrm{~kb}$ for budding yeasts. Despite the apparent lack of conservation, we are beginning to elucidate important functional regions of these RNAs.

Reprint requests to: Thomas R. Cech, Department of Chemistry and Biochemistry, University of Colorado, Boulder, CO 80309-0215, USA; e-mail: thomas.cech@colorado.edu.

Article and publication are at http://www.rnajournal.org/cgi/doi/ 10.1261/rna.5570803.
Phylogenetic analysis has greatly aided the understanding of telomerase RNA secondary structure in ciliates and vertebrates (Romero and Blackburn 1991; Lingner et al. 1994; McCormick-Graham and Romero 1995; Chen et al. 2000). In these two classes of organisms, a large phylogeny of sequences and relatively short RNAs facilitated comparative sequence analysis, leading to conserved core secondary structure predictions. These two core structures have striking similarities to each other, demonstrating that despite primary sequence divergence and differences in length, the important structural determinants of telomerase RNA function may in actuality be quite similar (Chen et al. 2000).

Secondary structure determination for yeast telomerase RNAs has been more challenging, primarily because of their longer lengths and the small pool of known yeast RNA sequences. However, some functional elements have been determined for the Saccharomyces cerevisiae RNA. An Smprotein-binding site was found to be critical for RNA maturation and stability (Seto et al. 1999). Through genetic approaches, a stem-loop structure was shown to be important for interaction with the DNA repair protein $\mathrm{Ku}$ (Peterson et al. 2001). Deletion analysis revealed that about half of the 
RNA was dispensable for in vivo function, and coimmunoprecipitation studies showed that distinct regions for binding two different protein subunits reside within the essential region (Livengood et al. 2002).

Through the cloning and sequencing of four additional Kluyveromyces species budding yeasts, followed by sequence comparison with Kluyveromyces lactis, a conserved paired element adjacent to the template was found. Biochemical and genetic analyses showed that the element specifies a template boundary (Tzfati et al. 2000). Cloning of additional Kluyveromyces species sequences allowed comparison of telomerase RNAs from six Kluyveromyces species, which uncovered seven highly conserved regions, two of which form a pseudoknot element that is essential for telomerase function (Tzfati et al. 2003). Comparison of these RNAs with the telomerase RNA from $S$. cerevisiae revealed a conserved bulged-stem structure that is important for interaction with S. cerevisiae Estlp, a telomerase regulatory subunit (Seto et al. 2002).

In addition to the Estlp-binding bulged stem, we now report that a template-proximal paired element is also conserved between the Kluyveromyces species and S. cerevisiae. Mutagenesis of this structure showed that it is important for telomerase function in vivo and in vitro. Sequencing of telomeres from mutant strains showed that disruption of the paired element can result in read-through beyond the template, or an alteration in usage of sequence within the template. Furthermore, mutants of this paired element have aberrant in vitro telomerase polymerization activity, implying functions of the element beyond serving as a template boundary.

\section{RESULTS}

\section{Identification and characterization of a putative template-proximal paired element in $S$. cerevisiae telomerase RNA}

To identify potential secondary structures in S. cerevisiae telomerase RNA (TLC1 RNA), the $5^{\prime}$ region of the RNA was subjected to the computer folding program RNA mfold (Zuker et al. 1999). A 7-bp helical structure was predicted to form immediately $5^{\prime}$ of the template region (boldface in Fig. 1A). A similar element was previously identified in Kluyveromyces telomerase RNAs, and shown to be important for specification of the template boundary (Tzfati et al. 2000). The putative paired element in S. cerevisiae shares characteristics with the Kluyveromyces element, in that the pairing spans $>300 \mathrm{nt}$ and is almost immediately adjacent to the template (Fig. 1A). As shown in Figure 1A, the predicted paired element is located in a position where it could delimit the extent of primer extension by telomerase, perhaps by forming a simple physical barrier to elongation.

To test the importance of the putative paired element in
A
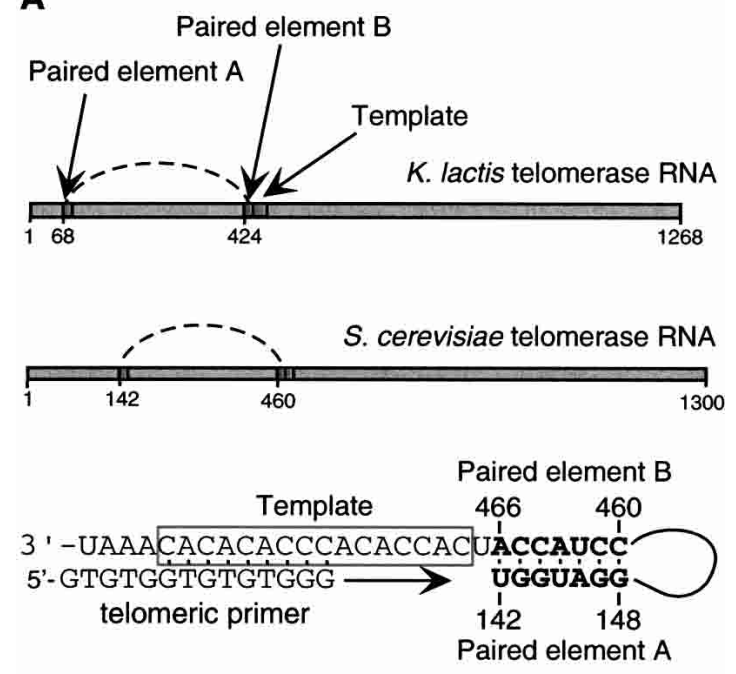

B

Disruption Mutant A (DMA)

3'-UAAACACACACCCACACCAQUACCAUCC

Disruption Mutant B (DMB)

3 '-UAAACACACACCCACACCAOUUGGUAGG
UGGUAGG

Disruption Mutant Compensatory (DMC)

3 ' -UAAA CACACACCCACACCAQUUUGGUAGGG

FIGURE 1. A conserved template-proximal paired element in telomerase RNAs from budding yeasts. $(A)$ A paired element located $5^{\prime}$ of the template region of Saccharomyces cerevisiae telomerase RNA was found by computer folding prediction. This long-range interaction is comprised of pairing between S. cerevisiae nucleotides 142-148 and 460-466 and resembles a similar element found in Kluyveromyces lactis telomerase RNA. (B) Mutations made in the S. cerevisiae telomerase RNA to test the predicted template-proximal paired element. Mutations were constructed in a CEN low-copy expression vector with TLC1 RNA expression driven from its endogenous promoter. Disruption Mutant A (DMA) replaced the sequence of paired element A with the sequence of paired element B. Disruption Mutant B (DMB) replaced the sequence of paired element $B$ with the sequence of paired element A. Disruption Mutant C (DMC) combined the DMA and $\mathrm{DMB}$ mutations to restore the pairing potential with a different sequence on both sides of the stem.

S. cerevisiae telomerase function, a series of mutations was designed to disrupt the stem or replace it with a compensatory sequence that restored the pairing potential of the two regions (Fig. 1B). Disruption Mutant A (DMA) replaces the sequence of paired element $A$ with the complementary sequence of paired element $B$, thereby disrupting the predicted base-pairing between these two elements. Disruption Mutant $\mathrm{B}$ (DMB) is similar to DMA in that the putative paired element is disrupted by replacement of the sequence of paired element $B$ with that of paired element $A$. 
Disruption Mutant Compensatory (DMC) contains both DMA and DMB block substitutions, thereby restoring the potential for base-pairing between the two regions while changing the sequence of each side of the helix. Computer folding of these mutants by RNA mfold predicted that the mutants should disrupt or compensate for the pairing as designed (data not shown).

Yeast strains with impaired telomerase undergo telomere shortening, leading to senescence, the eventual cessation of cell population growth. To test the effect of the pairing element mutants on in vivo telomerase function, the mutants were transformed into a $\operatorname{tlcl} \Delta \mathrm{rad52^{- }}$ strain. The rad52 strain allowed for clear assessment of defects in telomerase activity by disabling the alternative recombination pathway for telomere maintenance (Lundblad and Blackburn 1993). The complementing LYS2marked TLC1 plasmid was shuffled out by growth on $\alpha$-aminoadipate, which is toxic in the presence of the LYS2 gene product. After loss of the wild-type TLC1 plasmid, strains containing wildtype TLC1, DMA, DMB, DMC, or an empty vector were assayed for growth and telomere length. Colony growth of the DMA, DMB, and DMC strains was indistinguishable from the wild type (Fig. 2A), whereas the empty vector control, as expected, began to senesce 50-75 generations after loss of the wildtype plasmid. Growth of the disruption mutants was assayed for nine restreaks, corresponding to $\sim 225$ generations.

Interestingly, despite their wild-type growth phenotype, the DMA and DMB strains had shortened telomeres (Fig. 2B). Telomeres in the DMA and DMB strains appeared to be maintained at a stably shortened length over at least 98 generations (Fig. 2B; only three dilutions shown, corresponding to 42 generations). Presumably this length, which was greater than that seen in the early stages of growth of the control strain lacking telomerase (Fig. 2B, vector lanes), was sufficient to confer wild-type growth. Short telomeres coupled with an absence of growth phenotype have been seen before in strains carrying other mutations in the $S$. cerevisiae

A

telomerase RNA (Prescott and Blackburn 1997; Seto et al. 1999) or in essential protein components (Friedman and

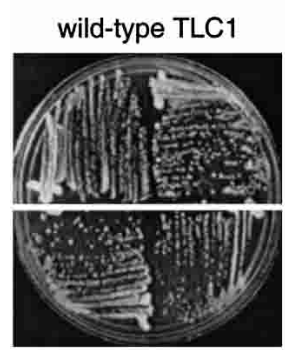

DMA

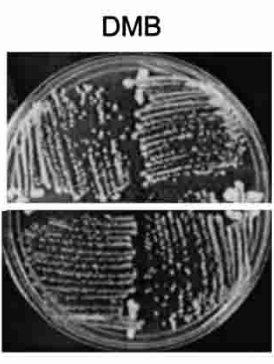

DMC

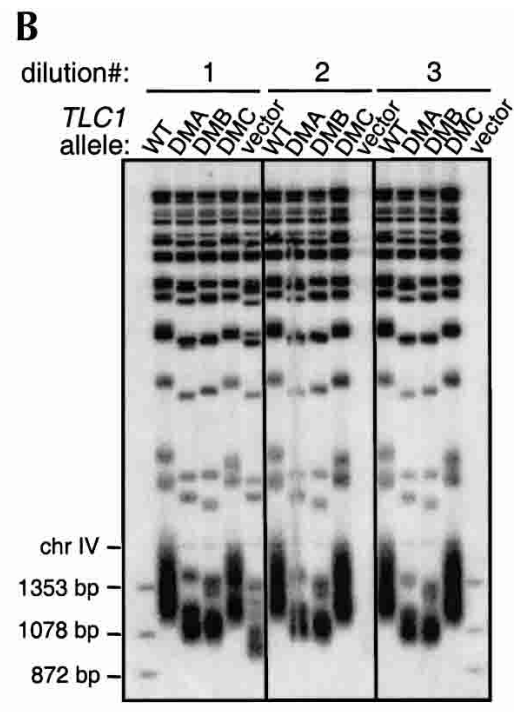

B

C
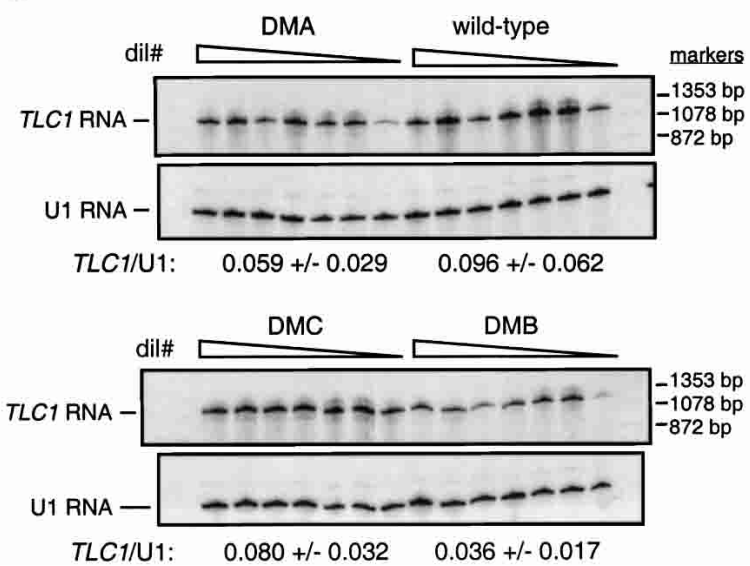

FIGURE 2. Analysis of growth, telomere lengths, and telomerase RNA levels of pairing element disruption mutants. (A) Growth of DMA, DMB, and DMC mutants compared with wild-type TLC1. DMA, DMB, DMC, wild-type TLC1, and an empty vector were transformed into a tlc1 $\mathrm{rad} 52^{-}$strain complemented with wild-type TLC1 on an LYS2 plasmid. After loss of the complementing wild-type TLC1 plasmid by growth on $\alpha$-aminoadipate, a single colony of each strain was restreaked to selective media. This senescence assay was carried out for nine restreaks, corresponding to $\sim 225$ generations. By the ninth restreak (shown), no senescence is observed. (B) Telomere length analysis of DMA, DMB, and DMC mutants compared with wild-type and empty vector. Strains were generated as described for $A$. After loss of the complementing wild-type TLC1 plasmid, a single colony was picked into liquid culture, diluted 200 -fold, then regrown to saturation. Four successive serial dilutions were performed. Genomic DNA was prepared from each saturated culture, corresponding to $\sim 42$ generations in total, digested with $X h o I$ to release terminal telomeric restriction fragments, resolved on an agarose gel, and analyzed by Southern blotting with probes to telomeric sequence and a region of Chromosome IV (chr IV). The markers shown were radiolabeled $\phi X 174-H a e I I I-d i g e s t e d ~ D N A$ fragments. $(C)$ Telomerase RNA levels of DMA, DMB, and DMC mutants. Single colonies of DMA, DMB, DMC, and wild-type TLC1 were picked into liquid culture and serially diluted seven times, as described for $B$, corresponding to $\sim 98$ generations of growth. Total RNA was prepared from these cultures and separated on a 4\% PAGE-7 M urea gel and Northern blotted; RNAs were visualized by hybridization with random-primed DNA probes to full-length TLC1 and U1 RNAs. Telomerase RNA levels were quantitated by PhosphorImager analysis. The TLC1 RNA signal was normalized to the U1 RNA signal in each lane. The mean of the TLC1/U1 ratios for the seven dilutions and its standard deviation are reported below each mutant analyzed. 
Cech 1999; Evans and Lundblad 2002). In contrast to the DMA and DMB mutants, the compensatory mutant (DMC) showed wild-type telomere length. Because the combination of two sets of deleterious mutations restored function, these results support the in vivo existence of the computer-predicted paired element. The compensatory mutant demonstrated that formation of the paired element is more important than the absolute sequence of the helix, because the DMC mutant was as effective as wild-type TLC1 in complementing a $t l c 1 \Delta$ strain.

Such severe telomere shortening as seen with DMA and DMB is not typical for block substitutions near the template. Other substitutions that replaced blocks of 4-8 nt in a different TLC1 region $3^{\prime}$ of the template showed little or no effect on telomere lengths (Seto 2002).

Telomerase RNA levels were assessed to determine whether the mutations in the paired element affected TLC1 RNA stability (Fig. 2C). Quantitation was performed by normalization of the TLC1 RNA signal to the U1 small nuclear RNA signal, which was used as an internal standard. Through 98 generations of growth, TLC1 RNA levels did not show any consistent upward or downward trend. Only the DMB mutation appeared to have a significant effect on TLC1 RNA levels: Its TLC1/U1 ratio was $0.036 \pm 0.017$, whereas the ratios for wild type (WT) and DMC were $0.096 \pm 0.062$ and $0.080 \pm 0.032$, respectively $(p=0.029$ for DMB compared with WT and $p=0.007$ for DMB compared with DMC, where $p$ is the probability that two populations are the same with respect to the variable tested, as determined by the Student's $t$-test). Although the mean value of the TLC1:U1 ratio for DMA $(0.059 \pm 0.029)$ was lower than that for WT, the difference was not statistically significant $(p=0.17)$. Thus, the approximately twofold reduction in RNA level in the DMB mutant may contribute to its short telomere phenotype.

The substitution of new sequence in an RNA may introduce new structural elements as well as disrupt the targeted element; therefore, we cannot assume that the overall structures of the DMA and DMB RNAs are identical. Structural differences may explain the difference in stability between these mutants.

\section{Impaired in vitro activity of paired element mutants}

To characterize more directly the effects of paired element mutations on telomerase function, in vitro telomerase activity assays were performed. Telomerase was immunopurified from extracts of strains expressing Protein-Atagged EST2 from its endogenous locus and wild-type or mutant TLC1 RNA from its endogenous promoter on a low-copy CEN plasmid. Bead-bound telomerase was incubated with a telomeric oligonucleotide primer, radiolabeled $\left[\alpha-{ }^{32} \mathrm{P}\right] \mathrm{dGTP}$, and either cold or radiolabeled TTP. The primer extension products were resolved on a polyacrylamide-urea gel. DMC had a level of activity and a pattern of products similar to those of wild type (Fig. 3A, cf. lanes 2 and 4). DMA and DMB both showed severely reduced levels of in vitro telomerase activity (Fig. 3A, lanes 3,5). The residual pattern of products was RNA-dependent, because preincubation of the beads with RNase A eliminated all activity (Fig. 3C, lanes 11-13; Fig. 4A, lanes 6-7), and is therefore attributed to telomerase rather than some other polymerization activity in the extracts. To visualize DMA products that were comparable in intensity to the weakest wild-type product, twofold to fourfold more immunoprecipitated DMA beads were typically used in the reaction. In vitro activity in the $\mathrm{DMB}$ mutant was barely visible above background and was not improved by increasing the amount of extract used in the immunoprecipitation or by optimization of the in vitro assay. Consequently, the in vitro characteristics of this mutant were not further investigated.

The lower in vitro activity of the DMA and DMB mutants could be explained if the mutant RNAs were defective in interaction with the catalytic subunit, Est $2 \mathrm{p}$. This possibility was tested by assaying the ability of these RNAs to coimmunoprecipitate with ProA-tagged Est2p. The RNA associated with IgG beads onto which the telomerase complex was immunoprecipitated was analyzed by Northern blotting using probes to TLC1 and U1 RNAs (Fig. 3B). The DMA and DMC mutants appeared to have little or no difference in coimmunoprecipitation with ProA-Est2p, compared with wild type. The DMB mutant, however, appeared to be less tightly associated with ProA-Est2p. Quantitation of three independent experiments showed that the signal of DMB bound was $16 \% \pm 8.7 \%$ of the signal of WT bound. In comparison, the signal of DMA bound was $\sim 95 \%$ that of WT bound (from two experiments). Therefore, the weaker association of the DMB mutant may explain the reduced level of in vitro telomerase activity. In contrast, the wildtype level of association of the DMA mutant indicated that the reduction in telomerase activity in this mutant was not caused by a weakened protein-RNA interaction or global misfolding, but rather reflects a deficiency in enzyme function.

The DMA mutant, although severely reduced in the levels of telomerase-extended products, was active enough to allow further in vitro characterization. The DMA mutant had two properties that differed from wild type: longer extension products and a changed pattern of those extension products (Fig. 3A). The longer products were unexpected, because the only nucleotides provided in this experiment were dGTP and TTP, and read-through synthesis should require dATP to copy the next RNA residue following the $5^{\prime}$ boundary of the template (see schematic, Fig. 3A). Therefore, such longer products, rather than being indicative of copying beyond the normal template $5^{\prime}$ boundary as described previously for $K$. lactis telomerase RNA mutants (Tzfati et al. 2000), could either reflect slippage synthesis, resulting in reiterative copying of a portion or portions of the normal template as reported for a template substitution 
A
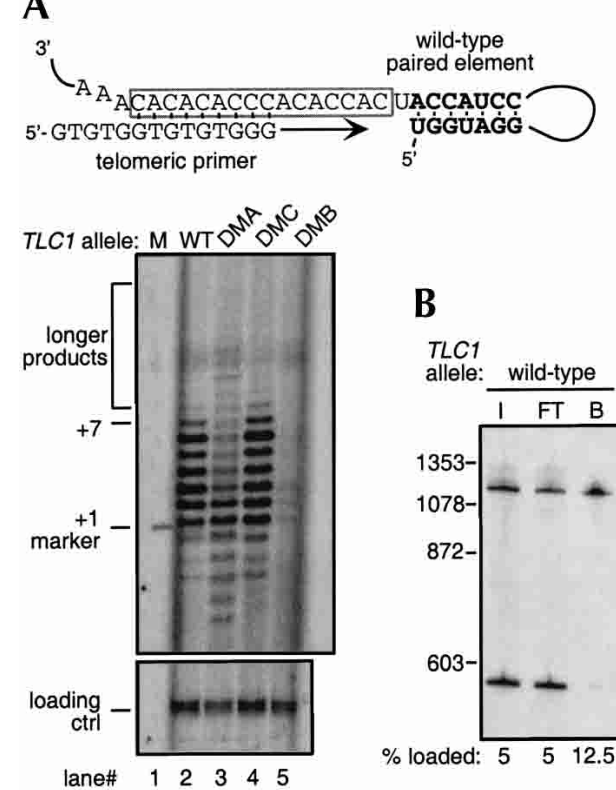

B
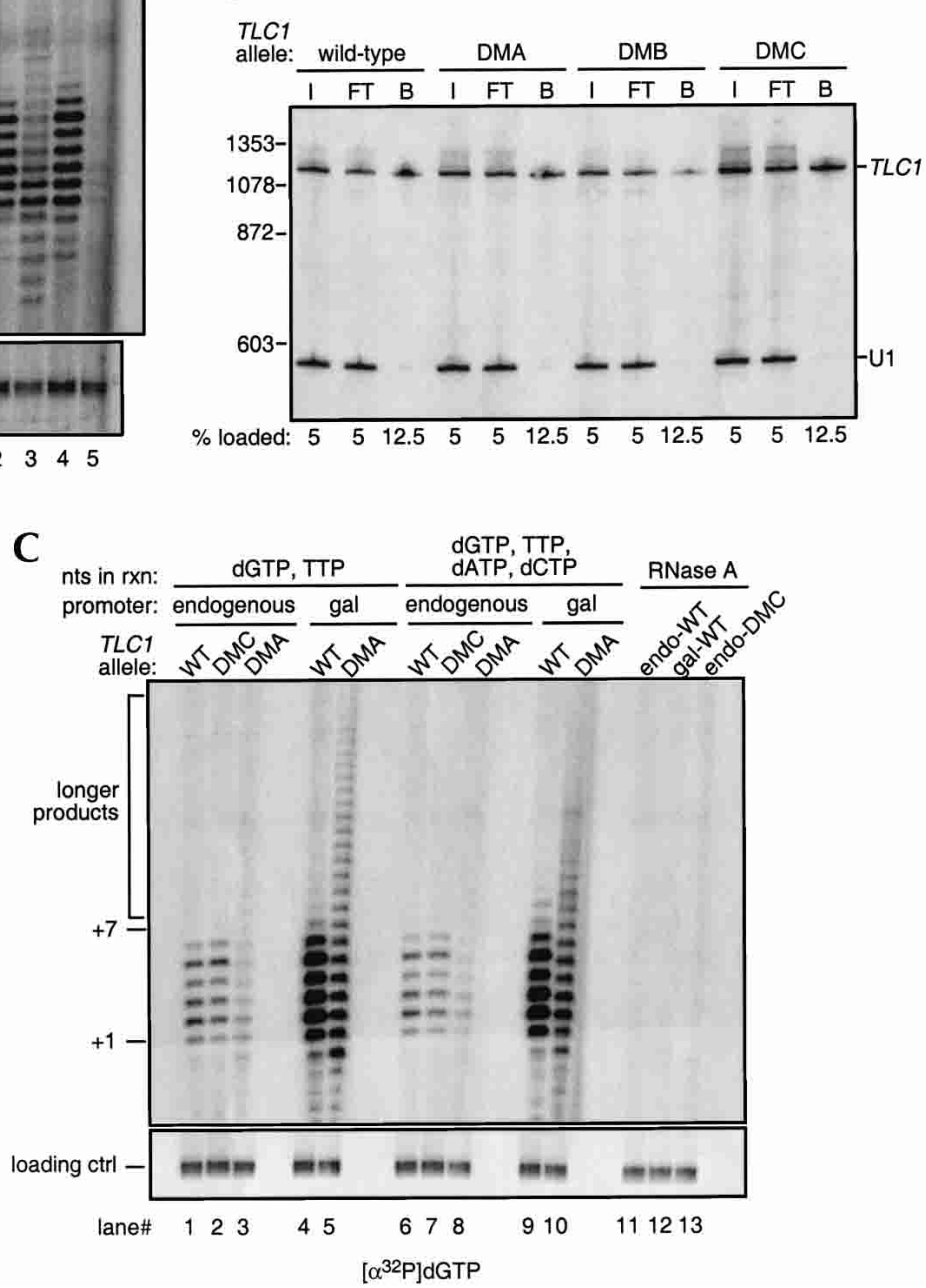

FIGURE 3. In vitro telomerase activity of paired element disruption mutants. (A) Telomerase activity immunopurified from wild-type, DMA, DMB, and DMC mutants. Extracts were prepared from a strain expressing ProA-tagged Est2p and wild-type, DMA, DMB, or DMC mutant TLC1 RNA. Extracts were then incubated with IgG beads to immunopurify telomerase. Beads were assayed for in vitro telomerase activity by incubation with $2.5 \mu \mathrm{M}$ telomeric primer, $100 \mu \mathrm{M}$ TTP, and $1.7 \mu \mathrm{M}\left[\alpha{ }^{32} \mathrm{P}\right] \mathrm{dGTP}$, with $5 \mu \mathrm{L}$ of wild-type beads, 2.5 $\mu \mathrm{L}$ of DMC, and $10 \mu \mathrm{L}$ each of DMA or DMB beads assayed. Telomerase products were resolved on a 12\% PAGE-7 $\mathrm{M}$ urea sequencing gel. (Loading ctrl) A control for product recovery: ${ }^{32} \mathrm{P} 5^{\prime}$-end-labeled 100 -nt oligonucleotide added immediately after the reactions were stopped and visualized on the same gel. (+1 marker) in lane $M$ was the 14-nt singlestranded telomeric primer extended by $1 \mathrm{nt}$ with the addition of $\left[{ }^{33} \mathrm{P}\right] \mathrm{ddTTP}$ and terminal deoxytransferase. (B) Coimmunoprecipitation of telomerase RNA mutants with ProA-Est2p. RNA coimmunoprecipitated with ProA-Est $2 p$ on IgG beads was isolated (as described for $A$ ), and analyzed by Northern blotting (as described for Fig. 2C). Blots were probed with radiolabeled TLC1 and U1 gene probes. (I) Input RNA; (FT) flowthrough from beads; (B) RNA bound to beads. The markers shown were radiolabeled $\phi X 174-H a e I I I-d i g e s t e d ~ D N A$ fragments. (\% loaded) Percentage of input, flowthrough, and bound RNAs loaded onto the gel. $(C)$ Telomerase activity in the presence of all four nucleotides, and the effect of overexpression of TLC1 RNA and ProA-Est2p. Reactions were performed in the absence or presence of both $100 \mu \mathrm{M}$ dATP and dCTP (lanes 1-5 and 6-10, respectively). In all cases, reactions contained $100 \mu \mathrm{M}$ TTP and $0.9 \mu \mathrm{M}\left[\alpha{ }^{-32} \mathrm{P}\right] \mathrm{dGTP}$. For the RNase A control reactions, beads were incubated with RNase A prior to the addition of the activity assay components. mutant of S. cerevisiae telomerase RNA (Prescott and Blackburn 1997), or increased processivity, allowing more than one round of template copying. Such multiround processive synthesis in vitro is not normally seen with wild-type yeast telomerase. In addition, the mobility of the products was reproducibly shifted slightly in the DMA mutant; this effect was seen most dramatically beginning at the +6 product and in more highly resolved electrophoresis gels (Fig. 3C, lanes 4,5). Because electrophoretic mobility of oligonucleotides is base-composition-dependent, the altered mobility indicated a different sequence for the DMA product.

Because of the weak activity of the DMA mutant, in vitro analysis of this mutant proved to be very challenging; we therefore constructed a $\operatorname{tlcl} \Delta$ strain in which both ProA-EST2 and TLC1 are expressed from a $G A L$ promoter on a plasmid. ProA-EST2 is also expressed from its endogenous locus. It has been previously shown that overexpression of both the RNA and reverse transcriptase components of telomerase results in a significant increase in in vitro activity (Teixeira et al. 2002). When the DMA mutant was overexpressed, we observed a significant boost in levels of in vitro activity (Fig. 3C, cf. lanes 3 and 5). The hallmarks of the DMA mutant-production of longer extension products and the shift in product mobility-were retained and became more obvious in the GAL-driven DMA expression strain. The DMB mutant activity was still too weak to allow further characterization (data not shown).

\section{Incorporation of noncognate nucleotides by paired element mutants in vitro}

This paired element in $S$. cerevisiae is found in the same location as the K. lactis paired element that showed longer extension products in vitro and in vivo, caused by disruption of the helical structure adjacent to the template boundary. We therefore reasoned that the addition of deoxyadenosine might allow the DMA mutant to extend beyond the S. cerevisiae template more efficiently, because templating by the sequence beyond the nor- 
A

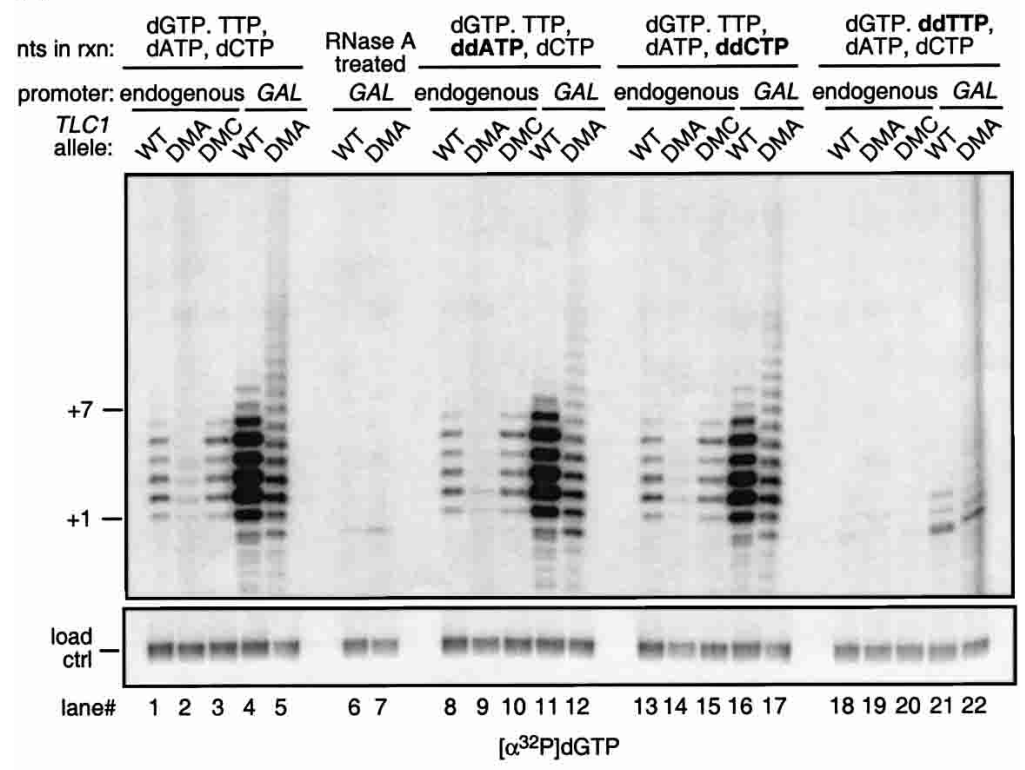

B

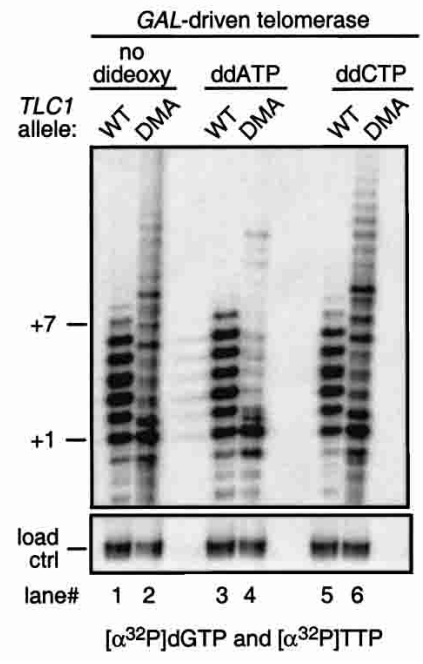

C

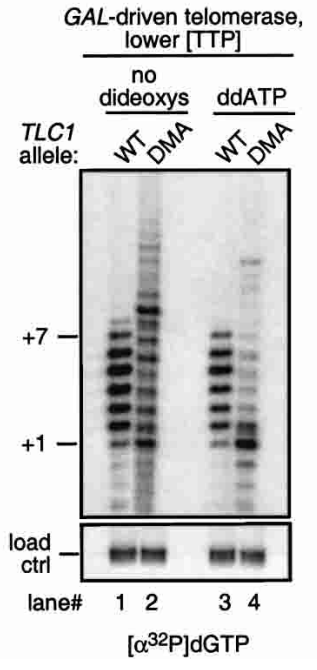

FIGURE 4. The effect of dideoxynucleotides on in vitro telomerase activity of paired element disruption mutants. (A) Activity of bead-bound telomerase in the presence of ddATP, ddCTP, and ddTTP. CEN- or GAL-expressed telomerase was immunopurified on IgG beads through ProA-Est2p, then assayed for in vitro telomerase activity in the presence of all four nucleotides. (Lanes 8-22) The corresponding dideoxynucleotide $(100 \mu \mathrm{M})$ was substituted for the deoxynucleotide. The other deoxynucleotides were present at $100 \mu \mathrm{M}$, and $\left[\alpha-{ }^{32} \mathrm{P}\right] \mathrm{dGTP}$ was added at $0.9 \mu \mathrm{M}$. The RNase A control reactions were performed as described for Figure 3B. (B) The effect of addition of ddTTP on DMA in vitro activity in the presence of $\left[\alpha-{ }^{32} \mathrm{P}\right] \mathrm{dGTP}$ and $\left[\alpha-{ }^{32} \mathrm{P}\right]$ TTP. $G A L$-driven wild-type and DMA telomerase were immunopurified on IgG beads. Assays were performed in the presence of all four deoxynucleotides (lanes 1,2), or with ddATP or ddCTP substituting for dATP or dCTP, respectively (lanes 3-6). In all cases, reactions were performed at $0.9 \mu \mathrm{M}\left[\alpha{ }^{32} \mathrm{P}\right] \mathrm{dGTP}$ and $\left[\alpha-{ }^{32} \mathrm{P}\right] \mathrm{TTP}$, and all other nucleotides were at $100 \mu \mathrm{M}$. (C) The effect of lowered TTP concentration on DMA in vitro activity. GAL-WT and GAL-DMA telomerase beads were incubated with $0.9 \mu \mathrm{M}\left[\alpha-{ }^{32} \mathrm{P}\right] \mathrm{dGTP}, 0.9 \mu \mathrm{M}$ cold TTP, $100 \mu \mathrm{M}$ dCTP, and 100 $\mu \mathrm{M}$ of dATP or ddATP.

mal template is predicted to require dATP in the absence of misincorporation (see schematic, Fig. 3A). The effect of dCTP was tested simultaneously.

Interestingly, the addition of dATP and dCTP had no apparent effect on the amount of longer extension products, although it did affect the mobility of the products- the +5 and +6 products of lanes 4 and 5 in Figure 3C more closely comigrated than those of lanes 9 and 10, which showed a larger mobility difference (Fig. 3C). This effect was reproducible over three independent experiments. Attempts to PCR-amplify, clone, and sequence the reaction products proved futile, as the excess of unextended primer interfered with the purification of extended products.

To assess whether the DMA mutant incorporated dATP or dCTP in the reaction products, assays were performed in the presence of ddATP or ddCTP. Polymerases cannot extend the dideoxy moiety; therefore, if ddATP or ddCTP is incorporated, shorter products would accumulate through chain termination. In reactions performed in the presence of $\left[{ }^{32} \mathrm{P}\right] \mathrm{dGTP}$, the indicated dideoxynucleoside triphosphate, and the other two deoxynucleoside triphosphates, we did not observe any effect of the dideoxy (Fig. 4A; e.g., cf. lanes 4,11 , and 16 or lanes 5,12 , and 17). These reactions were repeated in the presence of both $\left[{ }^{32} \mathrm{P}\right] \mathrm{dGTP}$ and $\left[{ }^{32} \mathrm{P}\right] \mathrm{TTP}$, to increase the intensity of weak bands. Interestingly, under these reaction conditions, we observed a strong accumulation of radiolabeled products at the +1 position in the presence of ddATP in the DMA mutant but not in wild type (Fig. 4B). These results indicated that some DMA products had incorporated a ddA at the +1 position.

At first glance, one may wonder why a primer extended by only cold ddATP would be radiolabeled. However, a yeast telomerase-associated exonuclease has been shown to digest the unextended primer, producing a radiolabeled +0 or shorter primer that will be subsequently extended by telomerase in vitro, resulting in products that are radiolabeled (Niu et al. 2000).

We did not observe the ddATP effect in the presence of only dGTP as the radiolabeled nucleotide, indicating two possibilities: first, the products containing adenosine at the +1 position are not visible unless labeled with $\left[{ }^{32} \mathrm{P}\right] \mathrm{TTP}$; or second, the $100 \mu \mathrm{M}$ cold TTP in the reactions shown in Figure 4A suppresses incorporation of ddATP, whereas $0.9 \mu \mathrm{M}\left[{ }^{32} \mathrm{P}\right] \mathrm{TTP}$ does not. To test the second hypothesis, reactions were performed with $0.9 \mu \mathrm{M}\left[{ }^{32} \mathrm{P}\right] \mathrm{dGTP}$, $0.9 \mu \mathrm{M} \mathrm{TTP}$, and $100 \mu \mathrm{M}$ dATP or ddATP. As shown in Figure $4 \mathrm{C}$, the ddATP effect of Figure $4 \mathrm{~B}$ was recapitulated under these reaction conditions. Therefore, it appears that 
at high TTP concentrations, the incorporation of ddATP by the DMA mutant is suppressed. Perhaps the DMA mutant has higher affinity for TTP, which is a natural $S$. cerevisiae telomerase substrate, than for ddATP. Thus, in the presence of high TTP concentrations, the enzyme might preferentially misincorporate TTP opposite uridines in the template, at positions where dATP or ddATP would otherwise be added. Alternatively, perhaps a second enzyme activity is dominant at high TTP concentration and masks the activity that would use dATP.

The DMA mutant produced bands at the +6 and +9 positions that were not seen with wild-type telomerase, and that disappeared specifically with ddATP but not ddCTP (Fig. 4B, cf. lanes 2 and 6 with lane 4; Fig. 4C, cf. lanes 2 and 4). In addition to the accumulation of a strong band at the +1 position, the presence of ddATP resulted in a faint pattern of longer extension products that culminate in a longer product at approximately the +11 or +12 position (Fig. $4 \mathrm{C}$, lane 4). This band appears to be telomerase-derived, as it does not appear in the wild-type products; it may reflect a product caused by extension beyond the conventional telomerase RNA template, consistent with our hypothesis that mutation of the paired element would disrupt the ability of the RNA to specify a template boundary. The faint intermediate bands may represent another telomerase activity that does not require $\mathrm{dATP}$.

\section{Sequence analysis of telomeric DNA from pairing element mutant strains}

To better understand the in vivo function of the templateproximal paired element, telomeric DNA from each paired element mutants was PCR-amplified, cloned, and sequenced. These sequences were compared with those from the wild-type strain sequenced in parallel. Most of the telomeres isolated from the CEN-DMA strain had normal telomeric sequence, although they were very short (24-99 nt for CEN-DMA compared with 108-199 nt for WT). However, in four of 13 clones, the telomeres were longer and revealed incorporation of sequence complementary to that of pairing element B (one clone shown in Fig. 5). Read-through of up to $8 \mathrm{nt}$ beyond the normal template was observed. These results indicate that at least some portion of the telomerase enzyme reads beyond the template to incorporate nontelomeric sequence. Interestingly, the long telomeres from the $G A L$-DMA strain had wild-type sequence, showing no incorporation of nontemplate sequence. We do not understand at present how overexpression of the mutant RNA would result in the normal sequence being incorporated.
The telomeres from the DMB mutant also had blocks of aberrant sequence, but these sequences do not appear to result from read-through beyond the template boundary. Instead, long stretches of TG dinucleotides were found at the telomeres of five of $15 C E N-D M B$ clones and nine of 12 $G A L-D M B$ clones. These strains had at least eight and up to 26 TG repeats, whereas in wild type, the longest stretch seen was seven repeats. As described in the Discussion, this mutant may use a small portion of the template in a repetitive fashion to generate these repeats.

\section{DISCUSSION}

The template-proximal paired element reported here represents the third of only three RNA secondary structure elements that have been experimentally verified for $S$. cerevisiae telomerase RNA. The other two secondary structures are a stem-loop involved in interaction with the Ku protein (Peterson et al. 2001) and a bulged stem necessary for binding the Estlp regulatory subunit of telomerase (Seto et al. 2002). As judged by the growth phenotype observed upon disruption of the template-proximal structure, this element is less important than the Est1p-binding site and comparable in importance to the $\mathrm{Ku}$ site. However, the templateproximal paired element directly affects the enzymatic activity of telomerase, whereas deletion of the $\mathrm{Ku}$ interaction site did not (Peterson et al. 2001).

The template-proximal paired element in S. cerevisiae resembles a similar structure found in Kluyveromyces species telomerase RNAs (Tzfati et al. 2000). This structure is also predicted by RNA mfold to form in telomerase RNAs from Saccharomyces mikatae and Saccharomyces bayanus (D. Zappulla, pers. comm.; sequences kindly provided by Manolis Kellis and Eric Lander, M.I.T.; Kellis et al. 2003). Disruption of this paired element in $K$. lactis resulted in extension 
beyond the template boundary, as shown by the dependence of the extension on dATP, the first nontemplate sequence, and other dNTPs expected to be incorporated, as well as sequence analysis of telomeric DNA synthesized in vivo by these mutant telomerases (Tzfati et al. 2000). Disruption of the $S$. cerevisiae paired element also results in the appearance of longer extension products in vitro, and some of these products are likely to be caused by template readthrough, because analysis of telomeric sequence from the CEN-DMA strain revealed incorporation of sequence complementary to RNA sequence found beyond the template. However, all of the in vitro products cannot be explained completely by read-through, because the appearance of longer products is not entirely dependent on the presence of dATP. It appears that we are observing overlapping activities of the DMA mutant. In addition to reading beyond the template boundary, the DMA mutant may also generate products through increased processivity from more efficient translocation and/or reiterative copying of a short region within the normal template. The sequencing of in vivo synthesized telomeres from the DMA strain supports this hypothesis: we have observed telomeres containing one full repeat followed by shorter, truncated repeats (Fig. 5).

The sequences of telomeric DNA isolated from the CENDMA mutant show that read-through is certainly occurring. However, the majority of cloned telomeres do not contain read-through sequences; perhaps most nontelomeric sequence is unstable and subsequently degraded, and there is likely to be selection for survival of yeast whose telomere sequences are normal enough to function in chromosome end protection.

In addition to generating unusually long products in vitro, the DMA mutant also produces short products with shifted mobilities compared to those of wild type. The changed mobility indicates incorporation of a different sequence within the first round of synthesis. A portion of these products disappears with the addition of ddATP at low TTP concentration, indicating that some but not all of the products contain adenosine. We do not know the mechanism of this action, but it is possible that the DMA mutant telomerase sometimes uses a different region of the template to begin synthesis and/or uses a different template sequence entirely. The sequences of the in vivo generated telomeres show no evidence for such an activity, but this may be a difference between in vivo and in vitro enzyme function.

Analysis of telomeres from the DMB mutant reveals unusual incorporation of stretches of TG repeats. One explanation for this observation is that the DMB mutation generated an RNA secondary structure that not only disrupted the template-proximal paired element, but also rearranged the overall structure surrounding the template. This altered structure may render most of the template inaccessible, thereby constraining the reverse transcriptase to the $3^{\prime}$ end of the template $\left(5^{\prime}\right.$-CACACAC- $\left.3^{\prime}\right)$. This result demonstrates that RNA structure is an important determinant for template usage by the telomerase catalytic protein subunit.

Previously, S. cerevisiae telomerase RNA mutants that contained triplet nucleotide substitutions in the template region were characterized in vitro (Prescott and Blackburn 1997). Substituting the three nucleotides immediately $5^{\prime}$ of the template (which we predict at least partially disrupts the pairing element) resulted in a strong +8 product, with the dNTP and ddNTP dependencies expected for read-through copying of at least one residue beyond the template sequence. In addition, some products had altered mobilities, compared with products of the wild-type enzyme, similar to what we report here with the DMA mutant.

In summary, the $S$. cerevisiae template-proximal paired element is not essential but contributes to in vivo telomere maintenance. It appears to function by specifying the template boundary, as has been shown for K. lactis. In addition, the biochemical characteristics of the paired element mutant indicate that this RNA element may have additional functions in determining enzyme processivity and template sequence usage. Future work should reveal if these functions are provided directly by the RNA or, rather, through its interaction with a protein component such as the Est $2 p$ catalytic subunit.

\section{MATERIALS AND METHODS}

\section{Plasmid construction}

pTLC1-DMA, pTLC1-DMB, and pTLC1-DMC were constructed by introducing the appropriate mutated sequence into pSD107 (TRP, CEN, and TLC1 with endogenous promoter and terminator sequences; Seto et al. 1999). The DMA mutant sequence was inserted by ligation of an StuI-NcoI PCR fragment generated with primers bearing the mutated sequence. The DMB mutant was constructed with an NcoI-HpaI PCR fragment containing the mutant sequence. DMC was generated by triple ligation of the StuINcoI DMA and NcoI-HpaI DMB fragments into the StuI and HpaI sites of pSD107. pRS314- $P_{\mathrm{GAL1}}-T L C 1$ (TRP, CEN, GAL-TLC1) was a gift of J. Lingner, ISREC, Lausanne, Switzerland (Teixeira et al. 2002). pGAL-DMA, pGAL-DMB, and pGAL-DMC were constructed by subcloning the BsrGI to NotI fragment from pTLC1DMA, pTLC1-DMB, or pTLC1-DMC into the corresponding sites in pRS314- $P_{\mathrm{GAL1}}-T L C 1$. For ProA-Est2p overexpression, GALpKF412 was constructed by PCR amplification of the GAL promoter from $\mathrm{pRS} 314-P_{\mathrm{GAL1}}-\mathrm{TLC1}$ with flanking ClaI restriction sites and subcloned into the ClaI site of pKF412 (URA, $2 \mu, P R O A-$ EST2). pKF412 was a gift from K. Friedman, Vanberbilt University. Mutagenesis and DNA manipulations were confirmed by DNA sequencing across the PCR-amplified region.

\section{S. cerevisiae strains and manipulations}

TCy43 ( $\Delta t l c 1:: L E U 2$, rad52::LEU2, pl-TLC1-LYS2-CEN; Seto et al. 1999) or ProA-Est2-tlc1::LEU2 (containing pRS316/TLC1; Livengood et al. 2002) was transformed with pSD107, pAS500 (empty 
vector; Seto et al. 1999), pTLC1-DMA, pTLC1-DMB, pTLC1DMC, pRS314- $P_{\mathrm{GAL} 1}-T L C 1$, pGAL-DMA, pGAL-DMB, or pGALDMC. After growth on selective media, single colonies were picked to $\alpha$-aminoadipate or 5 -FOA, to shuffle out the complementing pTLC1-LYS2-CEN or pRS316/TLC1, respectively. Single colonies were then restreaked to medium lacking tryptophan for further analysis. ProA-Est2-tlc1::LEU2 strains containing pRS314- $P_{\mathrm{GAL}^{-}}{ }^{-}$ TLC1, pGAL-DMA, pGAL-DMB, or pGAL-DMC were subsequently transformed with GAL-pKF412.

\section{Measurements of telomere length}

Single colonies from the first restreak on -TRP medium after growth on $\alpha$-aminoadipate were picked into $5 \mathrm{~mL}$ of selective liquid culture. The culture was grown to saturation, then diluted 200-fold to inoculate a fresh $5-\mathrm{mL}$ culture. Saturated cultures were harvested, and genomic DNA was prepared using the DNA-Pure Yeast Genomic Kit (CPG Inc.). Half of the isolated DNA was digested with $\mathrm{XhoI}$, resolved on a $1.1 \%$ agarose gel, then transferred to Hybond $\mathrm{N}+$ (Amersham Pharmacia) by osmoblotting. DNA was visualized with random-primed radiolabeled probes to telomeric repeat sequences and a fragment of Chromosome IV.

\section{Northern blot analysis of TLC1 RNA levels}

For quantitation of TLC1 RNA levels over time, 5-mL cultures were grown and diluted as described for telomere length analysis. Total RNA was prepared from cells using hot phenol extraction (Chapon et al. 1997). Approximately $15 \mu \mathrm{g}$ of total RNA was loaded onto a 4\% PAGE-7 M urea gel, then transferred to Hybond $\mathrm{N}+$ (Amersham Pharmacia) by electroblotting. RNAs were analyzed with random-primed probes to the TLC1 and U1 RNA genes. Quantitation was performed by PhosphorImager analysis using Imagequant software. $P$ values were calculated using a Webbased Student's $t$-test program (http://www.physics.csbsju.edu/ stats/t-test.html).

\section{Immunoprecipitation of telomerase and coimmunoprecipitation of TLC1 RNA}

Extracts were prepared from ProA-Est2-tlc1::LEU2 strains containing pSD107, pTLC1-DMA, pTLC1-DMB, pTLC1-DMC, pRS314- $P_{\text {GAL1 }}-T L C 1$, pGAL-DMA, pGAL-DMB, or pGAL-DMC as previously described (Livengood et al. 2002), followed by clarification at $16,000 \mathrm{~g}$. Extract protein concentrations were determined by Bradford assay. Approximately $1 \mathrm{mg}$ of total protein was added to $20 \mu \mathrm{L}$ of packed IgG beads (Amersham Pharmacia), then rotated end-over-end at $4^{\circ} \mathrm{C}$ for $6 \mathrm{~h}$, followed by three washes in extract buffer, followed by two washes in $10 \mathrm{mM}$ Tris- $\mathrm{HCl}(\mathrm{pH}$ 7.5), $1 \mathrm{mM} \mathrm{MgCl}_{2}, 10 \%$ glycerol, $1 \mathrm{mM} \mathrm{DTT,} 10 \mathrm{U} / \mathrm{mL}$ RNasin (Promega), and $1 \mathrm{mM}$ PMSF. Beads were resuspended in the final wash buffer. To examine coimmunoprecipitation of TLC1 RNA with ProA-Est2p, RNA bound to IgG beads and from input and flowthrough extracts was isolated as described (Seto et al. 1999). RNA was subjected to Northern blot analysis as described above.

\section{In vitro telomerase activity}

Bead-bound telomerase was prepared as described above. Bead supernatant was removed with a Hamilton syringe and incubated with telomerase assay buffer $(40 \mathrm{mM}$ Tris- $\mathrm{HCl}$ at $\mathrm{pH} 7.5,50 \mathrm{mM}$ $\mathrm{NaCl}, 5 \%$ [v/v] glycerol, $0.5 \mathrm{mM}$ spermidine, $0.5 \mathrm{mM}$ DTT, 0.9 $\mu \mathrm{M}\left[\alpha{ }^{32} \mathrm{P}\right] \mathrm{dGTP}, 100 \mu \mathrm{M}$ TTP, $2.5 \mathrm{mM} \mathrm{MgCl}_{2}$, and $2.5 \mu \mathrm{M}$ telomeric oligonucleotides) at $30^{\circ} \mathrm{C}$ for $20 \mathrm{~min}$. For increased signal, $0.9 \mu \mathrm{M}\left[\alpha-{ }^{32} \mathrm{P}\right] \mathrm{TTP}$ substituted for the cold TTP. Variations to the telomerase assay conditions are described in the text and figure legends. Telomerase extension products were isolated and analyzed as described previously (Seto et al. 1999).

\section{Cloning and sequence analysis of telomere fragments}

Genomic DNA was prepared from wild-type, DMA, DMB, and DMC strains, as described (McEachern and Blackburn 1996). DNA was resuspended in TE containing $100 \mu \mathrm{g} / \mathrm{mL}$ DNase-free RNase $\mathrm{A}$ and incubated at $37^{\circ} \mathrm{C}$ for $15 \mathrm{~min}$, followed by phenol: chloroform:isoamylalcohol (25:24:1) extraction, chloroform:isoamylalcohol (24:1) extraction, and ethanol precipitation. The genomic DNA was ligated to an anchor oligonucleotide that was $5^{\prime}$-phosphorylated and $3^{\prime}$-amino-modified to enable the ligation of its $5^{\prime}$ end, but not its $3^{\prime}$ end, to genomic DNA. A typical $20 \mu \mathrm{L}$ ligation reaction contained $2 \mu \mathrm{g}$ of genomic DNA, 60 pmole of an anchor oligonucleotide ( $5^{\prime}$-TTTAGTGAGGGTTAATAAGCGGC CGCGTCGTGACTGGGAGCGC-3'), 50 mM Tris-HCl (pH 8.0), 12.5\% PEG 8000, $10 \mathrm{mM} \mathrm{MgCl}_{2}, 1 \mathrm{mM}$ hexamine cobalt chloride, $20 \mu \mathrm{M}$ ATP, $10 \mu \mathrm{g} / \mathrm{mL}$ BSA, $1 \mathrm{mM}$ DTT, and 10 units of T4 RNA ligase (New England Biolabs). The reaction was incubated at $37^{\circ} \mathrm{C}$ for $1.5 \mathrm{~h}$. The ligated DNA was then amplified by PCR using a subtelomeric primer (5'-GTTGCACGGCCGTAGCGAGAG-3') and a primer complementary to the anchor oligonucleotide $\left(5^{\prime}\right.$ CGACGCGGCCGCTTATTAACCCT-3'). For efficient amplification of the repetitive, G-rich telomeric sequence, a high amount of Taq polymerase and a long extension time were used. Typically, a $40-\mu \mathrm{L}$ reaction included $100 \mathrm{ng}$ of anchor-primer ligated genomic DNA, $0.5 \mu \mathrm{M}$ each primer, $0.2 \mathrm{mM}$ each dNTP, $1.5 \mathrm{mM} \mathrm{MgCl}_{2}$, and 3.2 units of Taq polymerase (Bio-X-Act, Bioline $\mathrm{GmbH}$ ). The amplification cycles included initial denaturation at $94^{\circ} \mathrm{C}$ for 2 min and 30 cycles of denaturation at $95^{\circ} \mathrm{C}$ for $30 \mathrm{sec}$, annealing at $62^{\circ} \mathrm{C}$ for $45 \mathrm{sec}$, and extension at $72^{\circ} \mathrm{C}$ for $120 \mathrm{sec}$ in a T-Gradient thermal cycler (Biometra). The amplified telomere fragments were extracted from an agarose gel, cloned into a TA vector, and sequenced using either M13 universal or M13 reverse primer from the side of the telomeric end (using the G-rich strand as the sequencing template worked best). Sequencing was performed at The Center for Genomic Technologies, Silberman Institute of Life Sciences, The Hebrew University of Jerusalem. For each strain analyzed, 8-15 clones were sequenced. For strains expressing the wild-type and DMC RNAs from either the CEN or GAL constructs, the average length of telomeric sequence retrieved was similar: 162 bp and 169 bp for CEN-WT and CEN-DMC, or 182 bp and 214 bp for $G A L-W T$ and $G A L$-DMC, respectively. For strains carrying $C E N-D M A$ or $C E N-D M B$, the telomeric length was similar to each other and much shorter than that of wild type: $81 \mathrm{bp}$ and $89 \mathrm{bp}$, respectively. For the GAL-DMB strain, the average telomere length was $108 \mathrm{bp}$, which is significantly shorter than that of wild type or DMC. In contrast, with an average length of $285 \mathrm{bp}$, the cloned telomeres from the GAL-DMA strain were much longer than the GAL-WT or GAL-DMC lengths. Both wildtype and mutant telomeres showed some minor incorporation of nontelomeric sequence ( $0-4 \mathrm{nt}$ per telomere), which may be from misincorporation by telomerase or an artifact from the automated 
sequence analysis. These nucleotides were disregarded in our analysis.

\section{ACKNOWLEDGMENTS}

We thank V. Lundblad, K. Friedman, and J. Lingner for generous gifts of plasmids and strains. We thank K. Förstemann for suggesting the use of GAL-expressed telomerase, and A. Berglund and other members of the Cech and Blackburn labs for fruitful discussions and encouragement. This work was supported by National Institutes of Health Grants GM28039 (to T.R.C.) and GM26259 (to E.H.B.), and United States-Israel Binational Science Foundation (BSF) Grant 2001065 (to Y.T.).

The publication costs of this article were defrayed in part by payment of page charges. This article must therefore be hereby marked "advertisement" in accordance with 18 USC section 1734 solely to indicate this fact.

Received March 28, 2003; accepted July 31, 2003.

\section{REFERENCES}

Blackburn, E.H. 2000. The end of the (DNA) line. Nat. Struct. Biol. 7: 847-849.

Chapon, C., Cech, T.R., and Zaug, A.J. 1997. Polyadenylation of telomerase RNA in budding yeast. RNA 3: 1337-1351.

Chen, J.-L., Blasco, M.A., and Greider, C.W. 2000. Secondary structure of vertebrate telomerase RNA. Cell 100: 503-514.

Evans, S.K. and Lundblad, V. 2002. The Est1 subunit of Saccharomyces cerevisiae telomerase makes multiple contributions to telomere length maintenance. Genetics 162: 1101-1115.

Friedman, K.L. and Cech, T.R. 1999. Essential functions of aminoterminal domains in the yeast telomerase catalytic subunit revealed by selection for viable mutants. Genes \& Dev. 13: 2863-2874.

Greider, C.W. and Blackburn, E.H. 1987. The telomere terminal transferase of Tetrahymena is a ribonucleoprotein enzyme with two kinds of primer specificity. Cell 51: 887-898.

Kelleher, C., Teixeira, M.T., Forstemann, K., and Lingner, J. 2002. Telomerase: Biochemical considerations for enzyme and substrate. Trends Biochem. Sci. 27: 572-579.

Kellis, M., Patterson, N., Endrizzi, M., Birren, B., and Lander, E.S. 2003. Sequencing and comparison of yeast species to identify genes and regulatory elements. Nature 423: 241-254.

Lingner, J., Hendrick, L.L., and Cech, T.R. 1994. Telomerase RNAs of different ciliates have a common secondary structure and a per- muted template. Genes \& Dev. 8: 1984-1998.

Livengood, A.J., Zaug, A.J., and Cech, T.R. 2002. Essential regions of Saccharomyces cerevisiae telomerase RNA: Separate elements for Estlp and Est2p interaction. Mol. Cell. Biol. 22: 2366-2374.

Lundblad, V. and Blackburn, E.H. 1993. An alternative pathway for yeast telomere maintenance rescues est1-senescence. Cell 73: 347360.

McCormick-Graham, M. and Romero, D.P. 1995. Ciliate telomerase RNA structural features. Nucleic Acids Res. 23: 1091-1097.

McEachern, M.J. and Blackburn, E.H. 1996. Cap-prevented recombination between terminal telomeric repeat arrays (telomere CPR) maintains telomeres in Kluyveromyces lactis lacking telomerase. Genes \& Dev. 10: 1822-1834.

Niu, H., Xia, J., and Lue, N.F. 2000. Characterization of the interaction between the nuclease and reverse transcriptase activity of the yeast telomerase complex. Mol. Cell. Biol. 20: 6806-6815.

Peterson, S.E., Stellwagen, A.E., Diede, S.J., Singer, M.S., Haimberger, Z.W., Johnson, C.O., Tzoneva, M., and Gottschling, D.E. 2001. The function of a stem-loop in telomerase RNA is linked to the DNA repair protein Ku. Nat. Genet. 27: 64-67.

Prescott, J. and Blackburn, E.H. 1997. Telomerase RNA mutations in Saccharomyces cerevisiae alter telomerase action and reveal nonprocessivity in vivo and in vitro. Genes \& Dev. 11: 528-540.

Romero, D.P. and Blackburn, E.H. 1991. A conserved secondary structure for telomerase RNA. Cell 67: 343-353.

Seto, A.G. 2002. "Functional elements in the Saccharomyces cerevisiae telomerase RNA." Ph.D. thesis, University of Colorado, Boulder, CO.

Seto, A.G., Zaug, A.J., Sobel, S.G., Wolin, S.L., and Cech, T.R. 1999. Saccharomyces cerevisiae telomerase is an Sm small nuclear ribonucleoprotein particle. Nature 401: 177-180.

Seto, A.G., Livengood, A.J., Tzfati, Y., Blackburn, E.H., and Cech, T.R. 2002. A bulged stem tethers Estlp to telomerase RNA in budding yeast. Genes \& Dev. 16: 2800-2812.

Teixeira, M.T., Förstemann, K., Gasser, S.M., and Lingner, J. 2002. Intracellular trafficking of yeast telomerase components. EMBO Rep. 3: 652-659.

Tzfati, Y., Fulton, T.B., Roy, J., and Blackburn, E.H. 2000. Template boundary in a yeast telomerase specified by RNA structure. Science 288: 863-867.

Tzfati, Y., Knight, Z., Roy, J., and Blackburn, E.H. 2003. A novel pseudoknot element is essential for the action of a yeast telomerase. Genes \& Dev. 17: 1779-1788.

Zuker, M., Mathews, D.H., and Turner, D.H. 1999. Algorithms and thermodynamics for RNA secondary structure prediction: A practical guide. In RNA biochemistry and biotechnology (eds. J. Barciszewski and B.F.C. Clark), pp. 11-43. Kluwer Academic Publishers, Dordrecht, NL. 

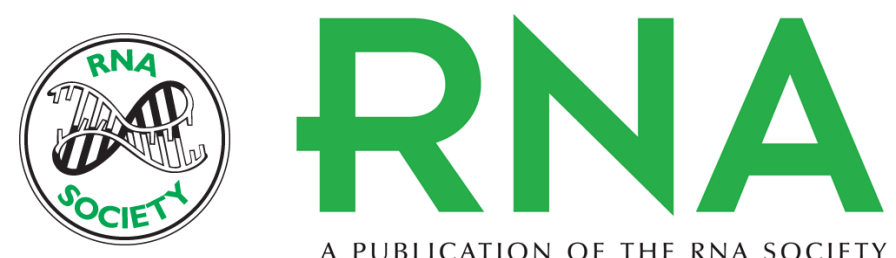

A PUBLICATION OF THE RNA SOCIETY

\section{A template-proximal RNA paired element contributes to Saccharomyces cerevisiae telomerase activity}

ANITA G. SETO, KFIR UMANSKY, YEHUDA TZFATI, et al.

RNA 2003 9: 1323-1332

References This article cites 22 articles, 11 of which can be accessed free at: http://rnajournal.cshlp.org/content/9/11/1323.full.html\#ref-list-1

License

Email Alerting Receive free email alerts when new articles cite this article - sign up in the box at the Service top right corner of the article or click here. 